\title{
Mathematical analysis of the effect of tectonic stress on the stability of underground tunnels
}

\author{
T. Hon* Y. H. Wu* $\quad$ B. Wiwatanapataphee ${ }^{\dagger}$ \\ C. X. Yang* $\ddagger$
}

(Received 28/10/05; revised 12 May 2007)

\begin{abstract}
We present a no-tension elastic-plastic model and a numerical method for analysing the stability of underground tunnels. The governing equations include equations of motion and a nonlinear constitutive equation. A finite element numerical scheme is developed to solve the problem taking into account the presence of tectonic stresses in rock masses. The numerical scheme is then used to study the effect of the orientation and magnitude of the tectonic stresses on the distribution of stresses and yielding zones around the tunnels.
\end{abstract}

${ }^{*}$ Dept. of Mathematics and Statistics, Curtin Univ. of Tech., WA, Australia. mailto: yhwu@maths . curtin. edu . au

$\dagger$ Dept. of Mathematics, Faculty of Science, Mahidol University, Bangkok, ThaILAnd. mailto:scbww@mahidol.ac.th

${ }^{\ddagger}$ School of Resources and Civil Engineering, Northeastern University, China.

See http://anziamj.austms.org.au/V47EMAC2005/Hon for this article, (c) Austral. Mathematical Soc. 2007. Published July 18, 2007. ISSN 1446-8735 


\section{Contents}

1 Introduction

C859

2 Constitutive Equations

C860

3 Boundary value problem and numerical scheme

C863

4 Numerical investigation

C864

5 Conclusions

C870

References

C871

\section{Introduction}

For the safety and efficiency of mining operations, it is important to analyse the behaviour of rock masses around underground tunnels and slopes during mining operations, and to recommend required course of actions based on the predictions. The course of actions may involve the installation of specified support systems and the adoption of a particular excavation sequence.

Over the last few decades, great effort has been made worldwide to construct proper mathematical models to study the stability of underground tunnels under various geo-mechanical and operational conditions. Various numerical methods such as the finite element method, the boundary element method and the limit equilibrium method have been widely used to simulate the behaviour of rock masses around underground tunnels during mining operations $[1,3,5,7,8,9,10,11,12,14]$. As rock mass is one of the most complex engineering materials and the stability of mine structures are generally determined by many factors, predictions of the stability of mine structures such as underground tunnels are extremely complicated. To pro- 
duce accurate predictions for a particular case, it is essential to construct a proper constitutive model for the rock mass and take into account all geomechanical conditions such as the orientation and magnitude of tectonic stresses.

In Western Australia, intact rock materials in most mines are fairly strong but rock masses are heavily jointed, faulted and are subjected to very high tectonic stresses $[2,6]$. For these kinds of rock masses, rock failure may occur by tension or plastic yielding or combination of both forms. If the rock mass fails by tension, the tensile strength in the tension failure zone becomes zero and the stresses are transferred to other places. Thus, to accurately describe the stability of the underground tunnels, it is necessary to model the heavily jointed rock mass as a no-tension elastic-plastic material. It is also important to consider the effect of tectonic stresses on stability of the tunnels. In this article, we construct a set of constitutive equations capable of simulating the no-tension behaviour and plastic yielding of the rock masses based on our previous work on granular materials [13], and then use the model to analyse the effect of tectonic stresses on the stability of the tunnels. In Section 2, the no-tension elastic-plastic constitutive equations for the rock masses are presented. In Section 3, the underlying boundary value problem is presented together with a numerical method for the solution of the problem. In Section 4, numerical results are presented to demonstrate the effect of tectonic stresses on the stability state of the rock masses around the underground tunnels.

\section{Constitutive Equations}

The rock mass considered is assumed to be a no-tension elastic-plastic continuum which yields according to Drucker-Prager yield criterion and permits no tension, thus

$$
F^{p}(\boldsymbol{\sigma})=\eta I_{1}+\sqrt{J_{2}}-K=0, \quad F^{t}=\sigma_{i}=0,
$$


where $F^{p}$ and $F^{t}$ are the Drucker-Prager yield criterion [4] and the tensile failure criterion, respectively; $I_{1}$ and $J_{2}$ are the first invariant and the second invariant of the deviatoric stress tensor, respectively; $\sigma_{i}$ is the principal stress component; and $\eta$ and $K$ are the materials constants determined by the angle of internal friction $\varphi$ and the cohesion strength $c$ of the material. The Drucker-Prager yield criterion in principal stress space is a right circular cone equally inclined to the principal stress axes as shown in Figure 1, and the no-tension condition cuts off all parts of the cone for which any principal stress is positive. The remaining part of the cone is a convex body as shown in Figure 1 and we refer to it by $O E L A$. Hence, the yield surface is the surface of the convex body OELA and all possible stress states lie on or inside the surface. The yield state depends on the location of the stress state point, and in this analysis the stress states are classified into four modes: (a) elastic state for which $F^{p}<0$ and $F^{t}<0$, corresponding to the regime inside the convex body $O E L A$; (b) elastic-plastic state for which $F^{p}=0$ and $F^{t}<0$, corresponding to the regime on the Drucker-Prager cone surface of the convex body $O E L A$ but not including the line formed by the intersection with the tensile cut-off planes; (c) elastic-tensile failure state for which $F^{p}<0$ and $F^{t}=0$, corresponding to the regime on the tension cut-off planes but excluding the intersection line with the Drucker-Prager cone; (d) elasticplastic tensile failure state for which $F^{p}=0$ and $F^{t}=0$, corresponding to the intersection line between the Drucker-Prager cone surface and the tension cut-off planes.

As any of the stress states (a)-(c) can be considered as a special case of (d), in the following, we first present the constitutive relation in (d) in detail. This derivation is based on the decomposition of the total elastic-plastic strain increment $d \varepsilon$ into an elastic component $d \varepsilon^{e}$, a plastic component $d \varepsilon^{p}$ and a component due to tensile yielding $d \varepsilon^{t}$, that is

$$
d \varepsilon=d \varepsilon^{e}+d \varepsilon^{p}+d \varepsilon^{t} .
$$

The elastic strain increment $d \varepsilon^{e}$ is simply related to the stress increment $d \sigma$ 


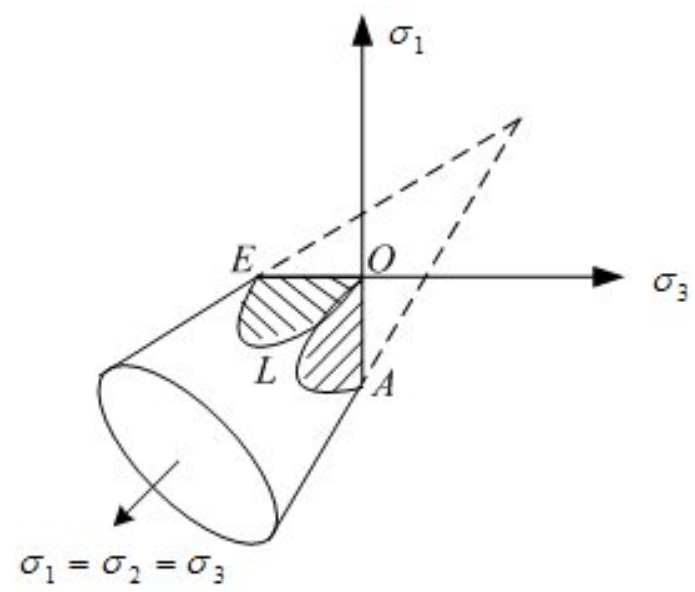

Figure 1: No-tension Drucker-Prager yield surface.

by Hooke's law

$$
d \varepsilon^{e}=\mathbf{D}_{e}^{-1} d \sigma,
$$

where $\mathbf{D}_{e}$ is the usual material elasticity matrix. Both the plastic strain increment $d \varepsilon^{p}$ and the tensile failure related strain increment $d \varepsilon^{t}$ are assumed to obey the associated plastic flow rule, thus

$$
d \varepsilon^{p}=\left\{\begin{array}{ll}
\lambda_{p} \frac{\partial F^{p}}{\partial \boldsymbol{\sigma}}, & \text { if } F^{p} \geq 0, \\
0, & \text { otherwise }
\end{array} \quad d \varepsilon^{t}= \begin{cases}\lambda_{t} \frac{\partial F^{t}}{\partial \boldsymbol{\sigma}}, & \text { if } F^{t} \geq 0 \\
0, & \text { otherwise }\end{cases}\right.
$$

where $\lambda_{p}$ and $\lambda_{t}$ are proportional constants. Following the procedure of $\mathrm{Wu}$ $\&$ Schmidt [13], we have

$$
\begin{aligned}
d \boldsymbol{\sigma} & =\left(\mathbf{D}_{e}-\frac{\mathbf{D}_{e} \mathbf{m}\left(\mathbf{m}^{T}-\alpha_{t}\right) \mathbf{D}_{e}}{\left(\mathbf{m}^{T}-\alpha_{t}\right) \mathbf{D}_{e} \mathbf{m}}-\frac{\mathbf{D}_{e} \mathbf{n}\left(\mathbf{n}^{T}-\alpha_{p}\right) \mathbf{D}_{e}}{\left(\mathbf{n}^{T}-\alpha_{p}\right) \mathbf{D}_{e} \mathbf{n}}\right) d \varepsilon \\
& =\left[\mathbf{D}_{e}-\mathbf{D}_{t}-\mathbf{D}_{p}\right] d \varepsilon=\left[\mathbf{D}_{e}-\mathbf{D}_{n}\right] d \varepsilon=\mathbf{D} d \varepsilon
\end{aligned}
$$

where

$$
\alpha_{p}=\frac{\mathbf{n}^{T} \mathbf{D}_{e} \mathbf{m} \mathbf{m}^{T}}{\mathbf{m}^{t} \mathbf{D}_{e} \mathbf{m}}, \quad \alpha_{t}=\frac{\mathbf{m}^{T} \mathbf{D}_{e} \mathbf{n} \mathbf{n}^{T}}{\mathbf{n}^{t} \mathbf{D}_{e} \mathbf{n}}, \quad \mathbf{m}=\frac{\partial F^{p}}{\partial \sigma}, \quad \mathbf{n}=\frac{\partial F^{t}}{\partial \sigma},
$$




$$
\mathbf{D}_{n}= \begin{cases}0, & \text { elastic state } \\ \mathbf{D}_{p}, & \text { elastic-plastic state } \\ \mathbf{D}_{t}, & \text { elastic-tensile failure state } \\ \mathbf{D}_{p}+\mathbf{D}_{t}, & \text { elastic-plastic-tensile failure state }\end{cases}
$$

\section{$3 \quad$ Boundary value problem and numerical scheme}

From the principles of continuum mechanics, the stress and displacement field in the rock masses are governed by the following boundary value problem,

$$
\begin{aligned}
& \frac{\partial \sigma_{j i}}{\partial x_{j}}+f_{i}=0 \quad \text { in } \Omega, \\
& \varepsilon_{i j}(\mathbf{u})=\frac{1}{2}\left(u_{i, j}+u_{j, i}\right) \quad \text { in } \Omega, \\
& \sigma_{i j}=D_{i j r s} \varepsilon_{r s} \quad \text { in } \Omega, \\
& u_{i}=0 \quad \text { on } \partial \Omega_{1} \\
& \sigma_{i j} n_{j}=\bar{t}_{i} \quad \text { on } \partial \Omega_{2},
\end{aligned}
$$

where we use the index notation with repeated literal indices representing summation over the index range, $\partial \Omega=\partial \Omega_{1} \cup \partial \Omega_{2}$ is the boundary of $\Omega$ with $\partial \Omega_{1}$ representing the fixed boundary and $\partial \Omega_{2}$ representing the prescribed stress boundary, $\boldsymbol{\sigma}=\sigma_{i j}$ and $\mathbf{u}$ are the stress tensor and displacement vector respectively, $\mathbf{D}=D_{i j r s}$ denotes the constitutive matrix as defined in Section $2, f_{i}$ is the body force and $\bar{t}_{i}$ is the surface traction on $\partial \Omega_{2}$ which is related to the virgin stress field for the excavation problem.

The variational boundary value problem corresponding to the above boundary value problem is as follows. Find $\mathbf{u} \in \mathbf{V}$ such that

$$
a(\mathbf{u}, \mathbf{v})=L(\mathbf{v}) \quad \text { for all } \mathbf{v} \in \mathbf{V},
$$


where

$$
\begin{aligned}
& a(\mathbf{u}, \mathbf{v})=\int_{\Omega}(\mathbf{B v})^{T} \mathbf{D}(\mathbf{B u}) d \Omega, \quad L(\mathbf{v})=\int_{\Omega} \mathbf{v}^{T} \mathbf{f} d \Omega+\int_{\partial \Omega_{2}} \mathbf{v}^{T} \overline{\mathbf{t}} d s \\
& \mathbf{V}=\left\{\mathbf{v} \mid \mathbf{v} \in\left[H^{1}(\Omega)\right]^{2}, \mathbf{v}=0 \text { on } d \Omega_{1}\right\}, \quad \mathbf{B}=\left[\begin{array}{cc}
\frac{\partial}{\partial x} & 0 \\
0 & \frac{\partial}{\partial y} \\
\frac{1}{2} \frac{\partial}{\partial y} & \frac{1}{2} \frac{\partial}{\partial x}
\end{array}\right] .
\end{aligned}
$$

The Galerkin finite element procedure generates the discretised system of equations

$$
\mathbf{K U}=\mathbf{F},
$$

where $\mathbf{K}=\left[k_{i j}\right]$ and $\mathbf{F}=\left[f_{i}\right]$ with

$$
k_{i j}=a\left(\Phi_{i}, \Phi_{j}\right), \quad f_{i}=L\left(\Phi_{i}\right),
$$

in which $\{\Phi\}_{i=1}^{N}$ are the basis functions of $\mathbf{V}$.

\section{$4 \quad$ Numerical investigation}

To analyse the effect of tectonic stresses on the stability of underground tunnels, we consider the excavation of two underground tunnels under plane strain condition as shown in Figure 2. The finite element mesh used in the analysis is shown in Figure 3.

To model the excavation process of a tunnel, the interaction between the unexcavated rock in the tunnel with its surrounding is replaced by an equivalent surface traction condition as show in Figure 4. This surface traction is then incrementally removed from the system to model the excavation process. The effect of removing $\underline{f}$ from the system is equivalent to applying a surface traction with the same magnitude but in the opposite direction in the system. 


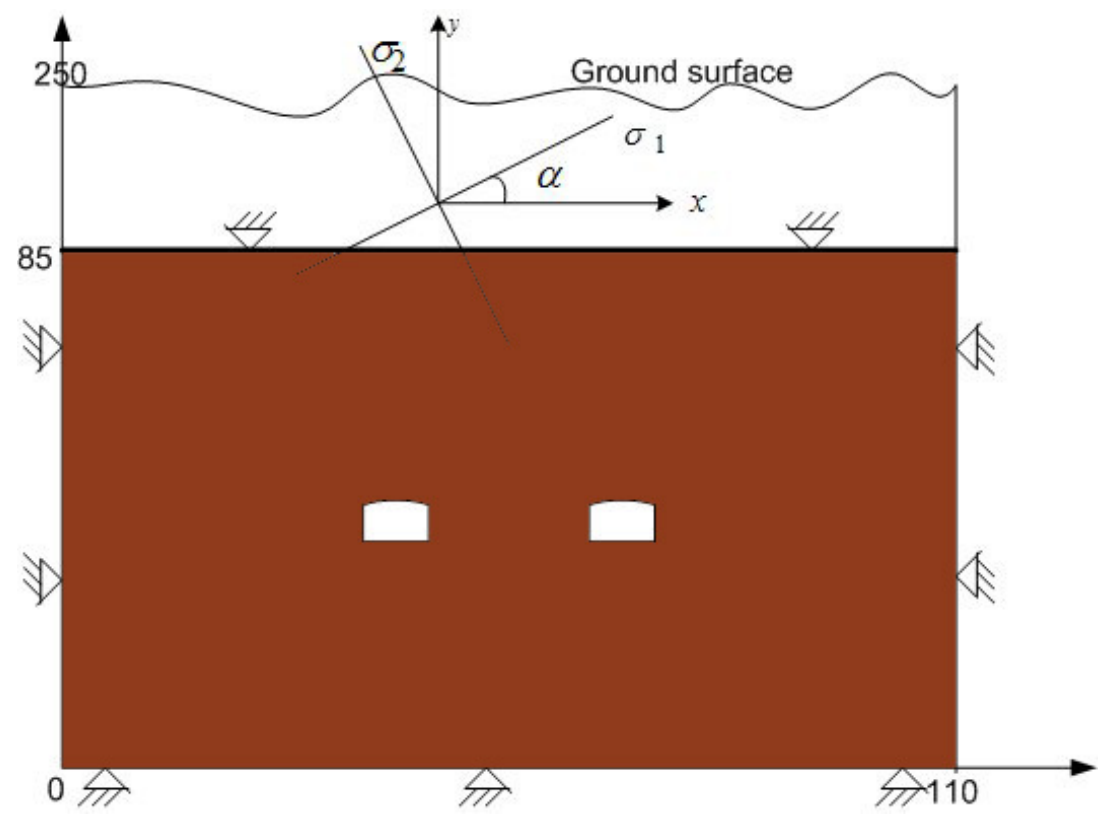

Figure 2: Computational region. 


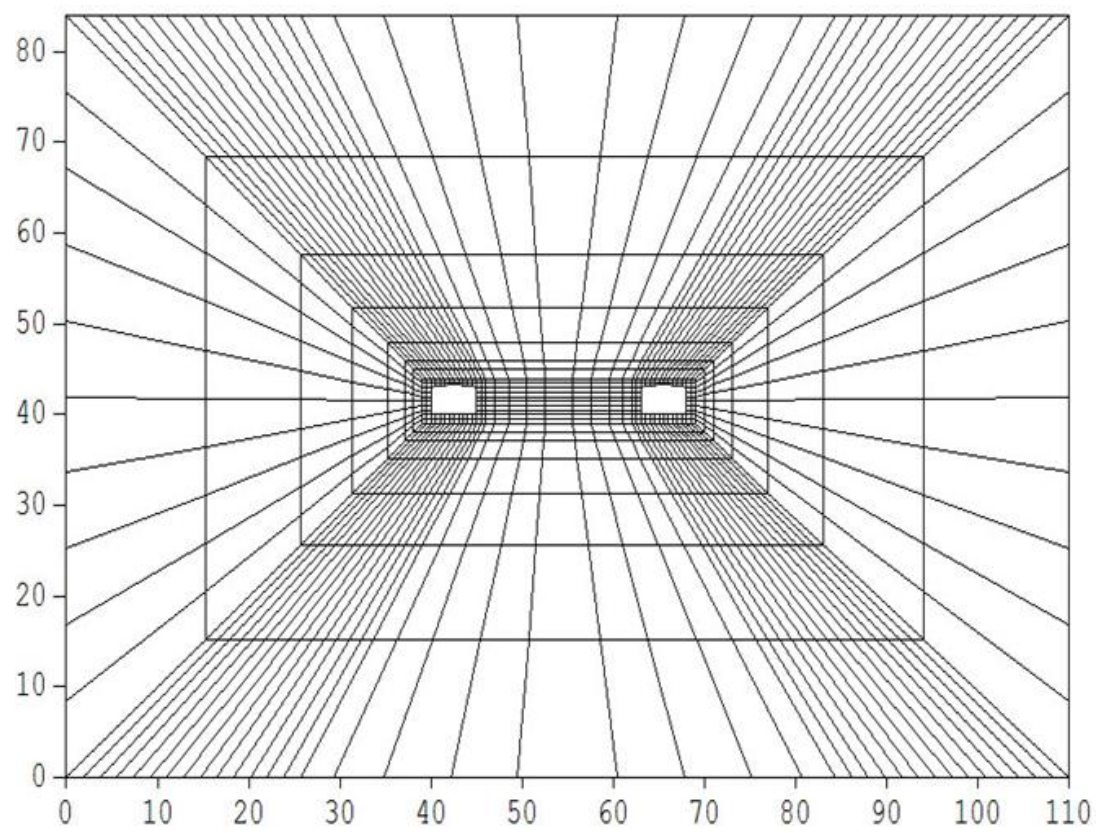

FiguRE 3: Finite element mesh.

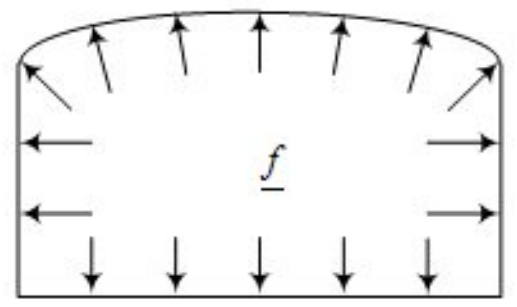

FiguRE 4: Virgin stress on the tunnel boundary 
The virgin stress field is taken to be

$$
\sigma_{2}=-\gamma H, \quad \sigma_{1}=-\left(\sigma_{T}+\frac{1}{2} \gamma H\right),
$$

where $\gamma=28 \mathrm{KN} / \mathrm{m}^{3}, H$ is the depth from the ground surface, $\sigma_{T}$ denotes the tectonic stress, $\sigma_{1}$ is the maximum principal stress which is inclined at an angle $\alpha$ to the horizontal as shown in Figure 2. In this study, we consider the effect of the magnitude of $\sigma_{T}$ and the effect of the angle $\alpha$ on the stability state of the rock mass around the tunnels. The value of $\sigma_{T}$ is taken to be 0 , 1.5 MPa, $3 \mathrm{MPa}, 6 \mathrm{MPa}$, while the value of $\alpha$ is taken to be $0^{\circ}, 5^{\circ}, 10^{\circ}$ and $15^{\circ}$. Other system parameter values are: Young's modulus $E=6.9 \times 10^{5} \mathrm{MPa}$, Poisson ratio $\nu=0.25$, cohesion $=2.0 \mathrm{MPa}$, internal friction angle $=23^{\circ}$ and the tensile strength $=0.1 \mathrm{MPa}$.

Figure 5 shows the distributions of maximum principal stresses versus distance from the tunnel along the line $\mathrm{AB}$ as shown in the figure. A significant relaxation of stress occurs near the edge of the tunnels. The angle $\alpha$ slightly affects the magnitude of the stresses along the examined locations.

The contour plots in Figures 6 and 7 show the distributions of the principal stresses around the tunnels for $\sigma_{T}=1.5 \mathrm{MPa}$ and $6 \mathrm{MPa}$ respectively. The angle $\alpha$ affects the distribution of maximum principal stresses very significantly. Compared with the results for $\alpha=0$, the maximum principal stresses for $\alpha=15$ are larger. The high compressive stress zone for $\alpha=15$ is located near the left corner of the roof and the right corner of the floor. By comparing the results in Figures 6 and 7, the magnitudes of the maximum principal stresses increase dramatically with increase in the tectonic stress.

Figure 8 shows the distribution of maximum principal stress in the rock around the left tunnel. For $\alpha=15$, a higher concentration of compressive stress occurs near the top left corner of the tunnel. This indicates that more support will be needed at this location. 

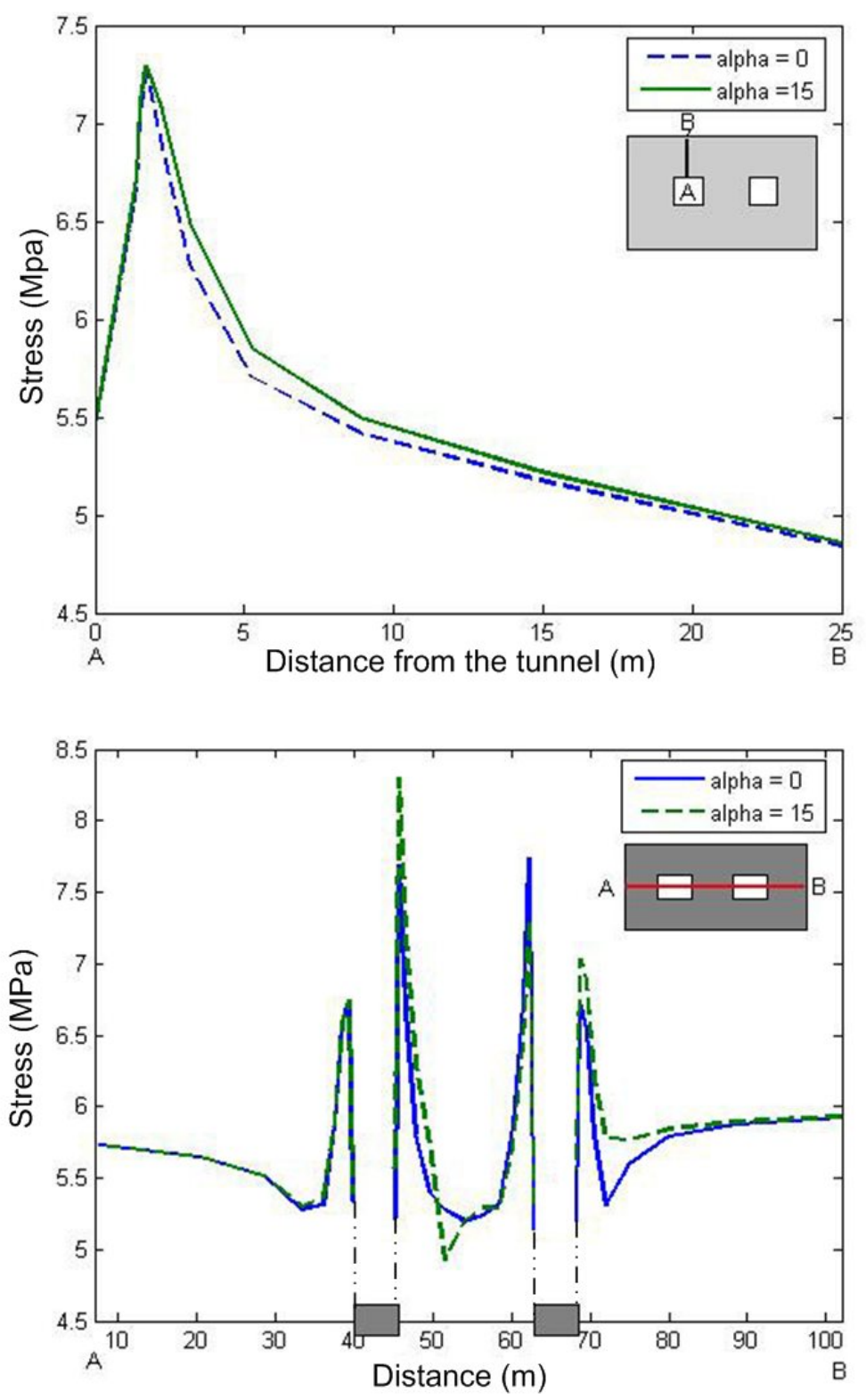

Figure 5: Maximum Stress distributions along AB. 

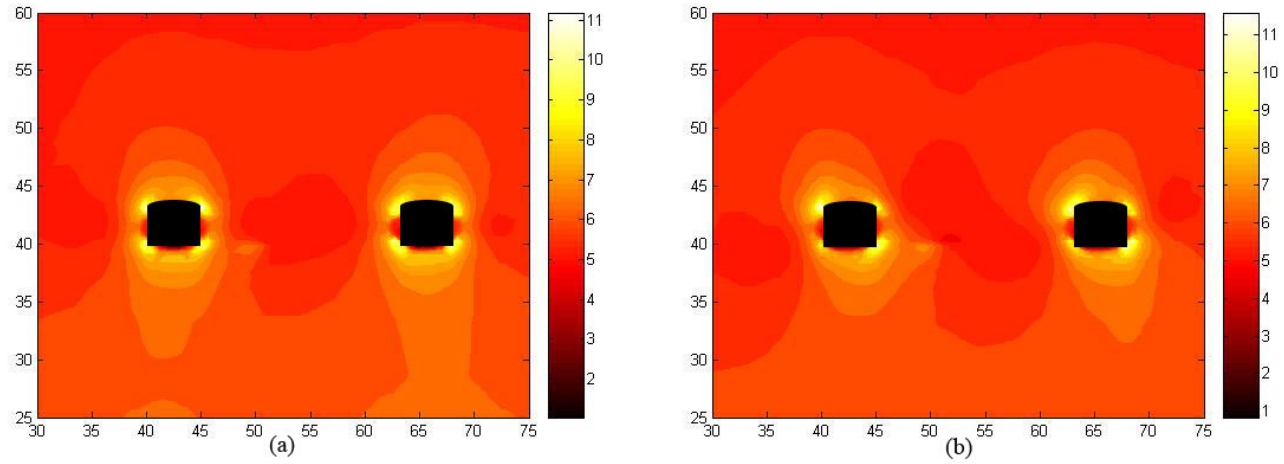

Figure 6: Maximum Stress with $\sigma_{T}=1.5$ for (a) $\alpha=0$, (b) $\alpha=15$.
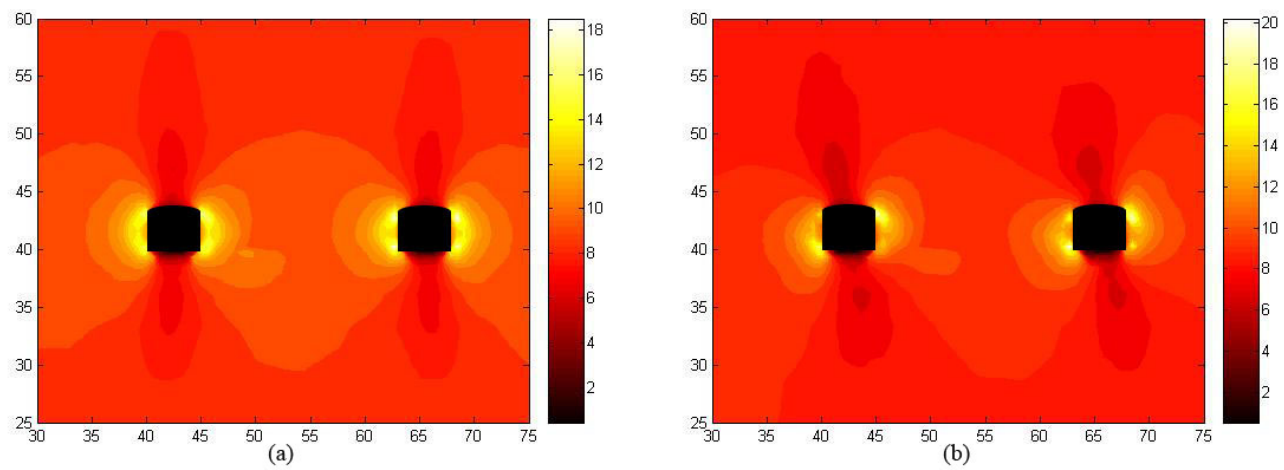

Figure 7: Maximum Stress with $\sigma_{T}=6$ for (a) $\alpha=0$, (b) $\alpha=15$. 


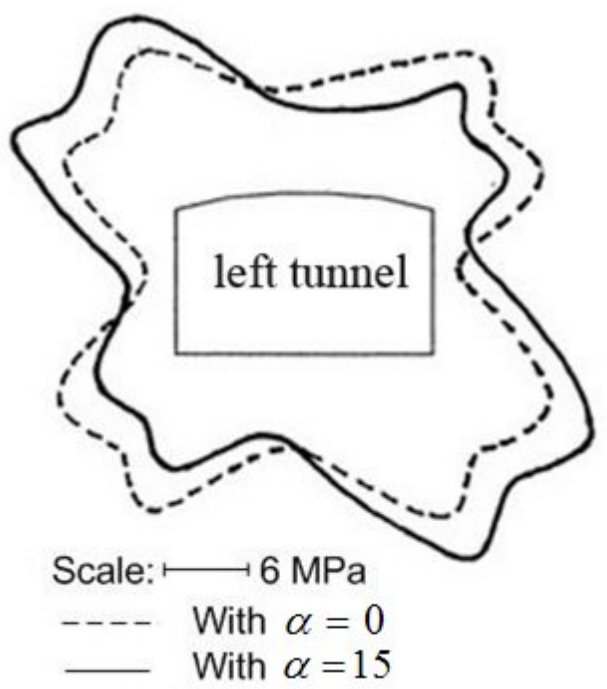

FiguRE 8: Distribution of Maximum principal stress around the tunnel

\section{Conclusions}

A finite element scheme based on the no-tension elastic-plastic constitutive model has been developed for the analysis of stress distribution and stability of rock masses around underground tunnels. Numerical investigations using the model show that the magnitude and orientation of tectonic stresses have significant effect on the distribution of stresses around the tunnels.

Acknowledgements: $\mathrm{Y} . \mathrm{H} . \mathrm{Yu}$ is grateful to the Australian Research Council, the Kalgoorlie Consolidated Gold Mines and the Barrick Darlot Gold Mine for the support of the project through an ARC linkage Project grant. 


\section{References}

[1] Beer G., and Poulsen B. A., "Efficient numerical modelling of faulted rock using the boundary element method", Int. Journal Rock Mechanic Mining Science and Geomechanic Absstract, 31(5), 1994, 485-506. C859

[2] Brown E. T., and Windsor C. R., "Near surface in-situ stresses in Australia and their influence on underground construction", The institution of Engineer Australia Tunnelling Conference' Sydney, September 1990. C860

[3] Cai M., and Horii H., "A contitutive model and FEM analysis of joint rock masses", Int. journal Rock Mechanic Mining Science and Geomechanic Abstract, 30(4), 1993, 351-359 C859

[4] Chen,W. F.,Contitutive equations for engineering material: Plastic and materials, Elsevier Science, London, 2, 1994. C861

[5] Diederichs M. S., and Kaiser P. K., "Rock instability and risk analysis in open stope mining design", Canadian Geotechical Journal, 33(3), 1996, 431-439. C859

[6] John Keogh, Plutonic Resource Limited Stress Mesurement, Australia Mining Consultances Pty Ltd, WA, AMC197199, 1998. C860

[7] Kawamoto, T., and Aydan O., " A review of numerical analysis of tunnels in discontinuous rock masses", International journal for numerical and analytical methods in geomechanics, 23,1999, 1377-1391. C859

[8] Ortiz, M., and Simo J. C., "An analysis of a new class of integration algroithms for elastoplastic constitutive relations", Int. Journal for numerical methods in engineering, 23, 1986, 353-366. C859 
[9] Sloan S. W., "Upper bound limit analysis using finite elements and linear programing", Int. Journal for Numerical and Analytical Methods in Geomechanics, 13, 1989, 263-282. C859

[10] Sourineni F. T., Tannant D. D., and Kaiser P. K., "Fault factor for stability graph method of open-stope design", Tran. Inst. Mining $\mathcal{E}$ Metallurgy Section B-Applied Earth Science,108, 1999, A92-A104. C859

[11] Valiappan S., and Pham T. D., "Elasto-plastic finite element analysis with fuzzy paramters", Int. Journal for Numerical Methods in Engineering, 38(4), 1995, 531-548. C859

[12] Wu Y. H., and Hii J., "Prediction of roadway stability in sub-level caving mines", 2nd Australian Conference computer application in mineral industry, University of Wollongong,1991, 159-164. C859

[13] Wu Y. H., and Schmidt L. C., "A boundary element method for prediction of silo pressure", Int. Journal of Computer and Structures, 45(2), 1992, 315-323. C860, C862

[14] Zienkiewicz, O. C., Valliappan, S., and King, I. P., "Stress Analysis of rocks as a no tension material", Geotechnique, 18, 1968, 55-66. C859 